\title{
Diastereoselective Cyclopropanation of Ketone Enols with Fischer Carbene Complexes
}

\author{
José Barluenga, ${ }^{*}$ Marcos G. Suero, Iván Pérez-Sánchez, and Josefa Flórez \\ Instituto Universitario de Química Organometálica "Enrique Moles" Unidad Asociada al CSIC, \\ Universidad de Oviedo, Julián Clavería 8, 33006 Oviedo, Spain \\ E-mail: barluenga@uniovi.es
}

\section{Supporting Information}

Starting materials. Carbene complexes $\mathbf{1 a},{ }^{1} \mathbf{1 b},{ }^{2} \mathbf{1 c},{ }^{1}$ and $\mathbf{1} \mathbf{d}^{3}$ were prepared according to the described procedures. The following silyl enol ethers, $(Z, E)$-4-trimethylsilyloxy-3-heptene $(Z: E, \quad 83: 17),{ }^{4} \quad(Z, E)-1-p h e n y l-2-t r i m e t h y l s i l y l o x y-1-p r o p e n e \quad(Z: E, \quad 98: 2),{ }^{5} \quad(E)-1-$ trimethylsilyloxy-1-cycloheptene, (E)-1-trimethylsilyloxy-1-cyclooctene, (Z)-4-methyl-3trimethylsilyloxy-2-pentene, ${ }^{6}$ were prepared according to a known procedure. ${ }^{7}$

\section{General Procedure for the Preparation of Ketone Lithium Enolates 2.}

Method A: To a solution of the corresponding silyl enol ether $(1.2 \mathrm{mmol})$ in THF $(10 \mathrm{~mL})$ at 0 ${ }^{\circ} \mathrm{C}$ was added butyllithium $(1.2 \mathrm{mmol}, 1.6 \mathrm{M}$ in hexane, $750 \mu \mathrm{L})$ and the mixture was stirred 30 min at $0{ }^{\circ} \mathrm{C}$ to complete formation of the lithium enolate.

Method B: $n$-Butyllithium $(1.2 \mathrm{mmol}, 1.6 \mathrm{M}$ in hexane, $750 \mu \mathrm{L})$ was added to a stirred solution of diisopropylamine $(1.2 \mathrm{mmol}, 172 \mu \mathrm{L})$ in THF $(2 \mathrm{~mL})$ at $0{ }^{\circ} \mathrm{C}$. After $15 \mathrm{~min}$, the solution was cooled to $-78{ }^{\circ} \mathrm{C}$ and the corresponding ketone $(1.2 \mathrm{mmol})$ in THF $(2 \mathrm{~mL})$ was added dropwise over $10 \mathrm{~min}$. The mixture was stirred at $-78^{\circ} \mathrm{C}$ for a further $30 \mathrm{~min}$ period to complete the enolization.

General Procedure for the Synthesis of Cyclopropanols 3. To a THF solution of the corresponding lithium enolate 2a-d $(1.2 \mathrm{mmol})$, prepared by method $A$ or $B$ as above indicated (see also Table 1), and cooled to $-78{ }^{\circ} \mathrm{C}$ was added carbene complex 1a (1 mmol, $312 \mathrm{mg}$ ) and THF $(10 \mathrm{~mL})$. The reaction mixture was stirred for $10 \mathrm{~min}$ while allowing the temperature to reach $-55^{\circ} \mathrm{C}$ and then stirred for a further $60 \mathrm{~min}$ period at $-55^{\circ} \mathrm{C}$. The initial dark red solution of the starting carbene complex turned orange-yellow gradually at $-55^{\circ} \mathrm{C}$. The cool bath was removed and the reaction mixture was allowed to get room temperature

\footnotetext{
${ }^{1}$ Fischer, E. O.; Kreiter, C. G.; Kollmeier, H. J.; Müller, J.; Fischer, R.D. J. Organomet. Chem. 1971, $28,237-$ 258.

${ }^{2}$ Brunner, H.; Koppelberger, J.; Fischer, E. O.; Lappus, M. J. Organomet. Chem. 1976, 112, 65-68.

${ }^{3}$ Zimmer, R.; Reissig, H-U. Journal fuer Praktische Chemie/Chemiker-Zeitung, 1998, 340, 755-756.

${ }^{4}$ Barluenga, J.; Montserrat, J. M.; Flórez, J.; García-Granda, S.; Martin, E. Chem. Eur. J. 1995, 1, $236-242$.

${ }^{5}$ Eames, J.; Coumbarides, G. S.; Suggate, M. J.; Weerasooriya, N. Eur. J. Org. Chem. 2003, 634-641.

${ }^{6}$ Xie, L.; Vanlandeghem, K.; Isember, K. M.; Bernier, C. J. Org. Chem. 2003, 68, 641-643.

${ }^{7}$ Heathcock, C. H.; Buse, C. T.; Kleschick, W. A.; Pirrung, M. C.; Sohn, J. E.; Lampe, J. J. Org. Chem. 1980, 45, 1066-1081.
} 
and stirred for 30 min to give a dark red-yellow mixture. Then the reaction mixture was cooled to $-78{ }^{\circ} \mathrm{C}$ and a solution of $2 \mathrm{~N} \mathrm{HCl}(2 \mathrm{~mL})$ and $\mathrm{H}_{2} \mathrm{O}(10 \mathrm{~mL})$ were sequentially added. The cool bath was removed and the reaction mixture was stirred for $30 \mathrm{~min}$ while allowing the temperature to reach room temperature. The resulting mixture was diluted with hexane/ethyl acetate, $10 / 1(110 \mathrm{~mL})$ and subjected to air oxidation under sunlight. After $\sim 2 \mathrm{~h}$ the yellow suspension was filtered through Celite and extracted with diethyl ether $(3 \times 10$ $\mathrm{mL}$ ). The organic layers were combined, dried over anhydrous $\mathrm{Na}_{2} \mathrm{SO}_{4}$ and concentrated in vacuo. The crude products were purified by flash column chromatography on silica gel (usually hexane/ethyl acetate, 20/1) to yield the corresponding cyclopropanols 3a (62\%), 3c (74\%), and 3d (79\%) as pure compounds and each one as a single diastereoisomer.

In two experiments the reaction mixture of carbene complex $1 \mathbf{a}$ and the appropiate lithium enolate 2a,b, prepared as above described, was stirred for only $5 \mathrm{~min}$ at $-78^{\circ} \mathrm{C}$ and then the cool bath was removed and the reaction mixture was allowed to reach room temperature with stirring for $45 \mathrm{~min}$. After acid hydrolysis and work up as above described diastereomerically pure cyclopropanols $3 \mathbf{a}(77 \%)$ and $\mathbf{3 b}(86 \%)$ were isolated.

(1S*,2S*,3R*)-3-Ethyl-2-methoxy-2-phenyl-1-propylcyclopropanol (3a). Colourless oil; $R_{\mathrm{f}}$ 0.22 (hexane:AcOEt, 9:1); ${ }^{1} \mathrm{H}$ NMR $\left(300 \mathrm{MHz}, \mathrm{CDCl}_{3}\right) \delta 0.75-0.88$ (m with $\mathrm{t}$ at 0.77 , $\left.{ }^{3} J(\mathrm{H}, \mathrm{H})=7.0 \mathrm{~Hz}, 5 \mathrm{H}\right), 1.13\left(\mathrm{t},{ }^{3} \mathrm{~J}(\mathrm{H}, \mathrm{H})=7.4 \mathrm{~Hz}, 3 \mathrm{H}\right), 1.40-1.76(\mathrm{~m}, 5 \mathrm{H}), 2.97(\mathrm{~s}, 1 \mathrm{H}), 3.19(\mathrm{~s}$, $3 \mathrm{H}), 7.29-7.36\left(\mathrm{~m}\right.$ with s at 7.34, 5H); ${ }^{13} \mathrm{C} \mathrm{NMR}\left(75 \mathrm{MHz}, \mathrm{CDCl}_{3}\right) \delta 14.1\left(\mathrm{CH}_{3}\right), 14.3\left(\mathrm{CH}_{3}\right)$, $14.9\left(\mathrm{CH}_{2}\right), 18.6\left(\mathrm{CH}_{2}\right), 30.2(\mathrm{CH}), 37.1\left(\mathrm{CH}_{2}\right), 55.4\left(\mathrm{CH}_{3}\right), 62.4(\mathrm{C}), 67.9(\mathrm{C}), 127.3(\mathrm{CH})$, $128.1(2 \times C H), 128.7(2 \times C H), 137.3(\mathrm{C})$; LRMS (70 eV, EI): m/z (\%): $234\left([\mathrm{M}]^{+}, 6\right), 233$ ([M$\left.\mathrm{H}]^{+}, 44\right), 131$ (39), 105 (100), 77 (52), 71 (86); HRMS (70 eV, EI) calcd for $\mathrm{C}_{15} \mathrm{H}_{22} \mathrm{O}_{2}$ [M$\mathrm{H}]^{+}:$233.1542, found: 233.1541 .

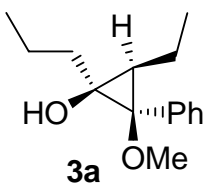

$\left(1 R^{*}, 2 S^{*}, 3 R^{*}\right)$-2-Methoxy-3-methyl-1,2-diphenylcyclopropanol (3b). Colourless oil; $R_{\mathrm{f}}$ 0.27 (hexane:AcOEt, 9:1); ${ }^{1} \mathrm{H}$ NMR $\left(400 \mathrm{MHz}, \mathrm{CD}_{2} \mathrm{Cl}_{2}\right) \delta 1.48\left(\mathrm{~d},{ }^{3} \mathrm{~J}(\mathrm{H}, \mathrm{H})=6.4 \mathrm{~Hz}, 3 \mathrm{H}\right)$, $2.23\left(\mathrm{q},{ }^{3} \mathrm{~J}(\mathrm{H}, \mathrm{H})=6.4 \mathrm{~Hz}, 1 \mathrm{H}\right), 3.35(\mathrm{~s}, 3 \mathrm{H}), 3.70($ broad s, $1 \mathrm{H}), 7,13-7.40(\mathrm{~m}, 10 \mathrm{H}) ;{ }^{13} \mathrm{C} \mathrm{NMR}$ $\left(100 \mathrm{MHz}, \mathrm{CD}_{2} \mathrm{Cl}_{2}\right) \delta 5.7\left(\mathrm{CH}_{3}\right), 22.8(\mathrm{CH}), 55.6\left(\mathrm{CH}_{3}\right), 63.9(\mathrm{C}), 69,5(\mathrm{C}), 126.4(\mathrm{CH}), 127.4$ (CH), $127.5(2 \times C H), 127.9(2 \times C H), 129.2(2 \times C H), 136.3(\mathrm{C}), 139.0(\mathrm{C})$; LRMS (70 eV, EI): m/z (\%): $254\left([\mathrm{M}]^{+}, 1\right), 253\left([\mathrm{M}-\mathrm{H}]^{+}, 6\right), 105$ (100), 77 (58); HRMS (70 eV, EI) calcd for $\mathrm{C}_{17} \mathrm{H}_{18} \mathrm{O}_{2}[\mathrm{M}-\mathrm{H}]^{+}: 253.1220$, found: 253.1228 .

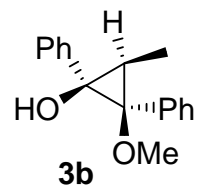

$\left(1 R^{*}, 7 R^{*}, 8 R^{*}\right)$-8-Methoxy-8-phenylbicyclo[5.1.0]octan-1-ol (3c). Colourless oil; $R_{\mathrm{f}} 0.25$ (hexane:AcOEt, 3:1); ${ }^{1} \mathrm{H}$ NMR $\left(400 \mathrm{MHz}, \mathrm{CD}_{2} \mathrm{Cl}_{2}\right) \delta 0.62-0.71(\mathrm{~m}, 1 \mathrm{H}), 0.95(\mathrm{qt}, J(\mathrm{H}, \mathrm{H})=$ 13.2, $3.5 \mathrm{~Hz}, 1 \mathrm{H}), 1.02(\mathrm{dd}, J(\mathrm{H}, \mathrm{H})=13.6,1.2 \mathrm{~Hz}, 1 \mathrm{H}), 1.20(\mathrm{ddd}, J(\mathrm{H}, \mathrm{H})=7.2,6.5,0.6 \mathrm{~Hz}$, $1 \mathrm{H}), 1.39-1.50(\mathrm{~m}, 1 \mathrm{H}), 1.80-1.84(\mathrm{~m}, 1 \mathrm{H}), 3.22(\mathrm{~s}, 3 \mathrm{H}), 7.27\left(\mathrm{~d},{ }^{3} \mathrm{~J}(\mathrm{H}, \mathrm{H})=1.4 \mathrm{~Hz}, 2 \mathrm{H}\right), 7.29-$ $7.39(\mathrm{~m}, 3 \mathrm{H}) ;{ }^{13} \mathrm{C} \mathrm{NMR}\left(100 \mathrm{MHz}, \mathrm{CDCl}_{3}\right) \delta 26.1\left(\mathrm{CH}_{2}\right), 29.3\left(\mathrm{CH}_{2}\right), 29.4\left(\mathrm{CH}_{2}\right), 31.7\left(\mathrm{CH}_{2}\right)$, $33.9\left(\mathrm{CH}_{2}\right), 35.0(\mathrm{CH}), 54.4\left(\mathrm{CH}_{3}\right), 65.2(\mathrm{C}), 73.9(\mathrm{C}), 128.2(\mathrm{CH}), 128.3(2 \mathrm{xCH}), 132.1$ (2xCH), 133.9 (C); LRMS (70 eV, EI): m/z (\%): $232\left([\mathrm{M}]^{+}, 5\right), 231\left([\mathrm{M}-\mathrm{H}]^{+}, 16\right), 121(34)$, 105 (100), 77 (66); HRMS (70 eV, EI) calcd for $\mathrm{C}_{15} \mathrm{H}_{19} \mathrm{O}_{2}[\mathrm{M}-\mathrm{H}]^{+}: 232.1380$, found: 232.1373 . 


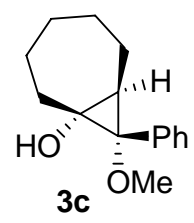

$\left(1 R^{*}, 8 R^{*}, 9 R^{*}\right)-9-M e t h o x y-9-p h e n y l b i c y c l o[6.1 .0]$ nonan-1-ol (3d). Colourless oil; $R_{\mathrm{f}} 0.10$ (hexane:AcOEt, 9:1); ${ }^{1} \mathrm{H}$ NMR $\left(400 \mathrm{MHz}, \mathrm{CDCl}_{3}\right) \delta 0.72(\mathrm{dq}, J(\mathrm{H}, \mathrm{H})=12.4,4.1 \mathrm{~Hz}, 1 \mathrm{H})$, $0.88(\mathrm{ddd}, J(\mathrm{H}, \mathrm{H})=14.4,13.3,3.8,1 \mathrm{H}), 1.19-1.28(\mathrm{~m}, 1 \mathrm{H}), 1.32-1.61(\mathrm{~m}, 4 \mathrm{H}), 1.64-1.75(\mathrm{~m}$, $4 \mathrm{H}), 1.79-1.89(\mathrm{~m}, 1 \mathrm{H}), 2.08-2.17(\mathrm{~m}, 2 \mathrm{H}), 3.08(\mathrm{bs}, 1 \mathrm{H}), 3.23(\mathrm{~s}, 3 \mathrm{H}), 7.33-7.37(\mathrm{~m}, 5 \mathrm{H}) ;{ }^{13} \mathrm{C}$ NMR $\left(100 \mathrm{MHz}, \mathrm{CDCl}_{3}\right) \delta 25.0\left(\mathrm{CH}_{2}\right), 25.9\left(2 \mathrm{xCH}_{2}\right), 26.9\left(\mathrm{CH}_{2}\right), 29.0\left(\mathrm{CH}_{2}\right), 30.0\left(\mathrm{CH}_{2}\right)$, $34.3(\mathrm{CH}), 55.3\left(\mathrm{CH}_{3}\right), 62.1(\mathrm{C}), 68.3(\mathrm{C}), 128.0(\mathrm{CH}), 128.2(2 \mathrm{xCH}), 131.5(2 \mathrm{xCH}), 134.8$ (C); LRMS (70 eV, EI): m/z (\%): 246 ([M] $\left.]^{+}, 22\right), 245$ ([M-H] $\left.]^{+}, 23\right), 189$ (79), 121 (94), 105 (100), 77 (79); HRMS (70 eV, EI) calcd for $\mathrm{C}_{16} \mathrm{H}_{21} \mathrm{O}_{2}$ [M-H] ${ }^{+}: 245.1533$, found: 245.1529.

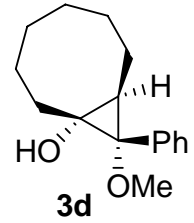

General Procedure for the Synthesis of Hydroxycyclobutanones 4. To a THF solution of the corresponding lithium enolate $\mathbf{2 c}, \mathbf{d}(1.2 \mathrm{mmol})$, prepared by method $B$ as above indicated and cooled to $-78{ }^{\circ} \mathrm{C}$ was added carbene complex 1a $(1 \mathrm{mmol}, 312 \mathrm{mg})$ and THF $(10 \mathrm{~mL})$. After $5 \mathrm{~min}$, the cool bath was removed and the reaction mixture was allowed to warm to room temperature and stirred for a further 45 min period. The initial dark red solution of the starting carbene complex turned orange-yellow gradually when the cool bath was removed and it turned dark red-yellow at room temperature. Then the reaction mixture was cooled to $78{ }^{\circ} \mathrm{C}$ and a solution of $2 \mathrm{~N} \mathrm{HCl}(2 \mathrm{~mL})$ and $\mathrm{H}_{2} \mathrm{O}(10 \mathrm{~mL})$ were sequentially added. The resulting mixture was diluted with hexane/ethyl acetate, $10 / 1(110 \mathrm{~mL})$ and subjected to air oxidation under sunlight. After $\sim 2 \mathrm{~h}$ the yellow suspension was filtered through Celite and extracted with diethyl ether $(3 \times 10 \mathrm{~mL})$. The organic layers were combined, dried over anhydrous $\mathrm{Na}_{2} \mathrm{SO}_{4}$ and concentrated in vacuo. The crude products were purified by flash column chromatography on silica gel (usually hexane/ethyl acetate, 20/1) to yield the corresponding hydroxycyclobutanones $\mathbf{4 a}(75 \%)$ and $\mathbf{4 b}(68 \%)$ as pure compounds and each one as a single diastereoisomer.

$\left(1 R^{*}, 7 R^{*}, 9 S^{*}\right)-7-H y d r o x y-9-m e t h o x y-9-p h e n y l b i c y c l o[5.2 .0]$ nonan-8-one (4a). Colourless solid; m.p. $180{ }^{\circ} \mathrm{C} ; R_{\mathrm{f}} 0.33$ (hexane:AcOEt, 9:1); ${ }^{1} \mathrm{H}$ NMR (400 MHz, $\left.\mathrm{CD}_{2} \mathrm{Cl}_{2}\right) \delta 1.30-1.45$ $(\mathrm{m}, 2 \mathrm{H}), 1.75-1.82(\mathrm{~m}, 1 \mathrm{H}), 1.84-2.10(\mathrm{~m}, 5 \mathrm{H}), 2.46(\mathrm{~s}, 1 \mathrm{H}), 2.52-2.59(\mathrm{~m}, 1 \mathrm{H}), 3.21(\mathrm{~s}, 3 \mathrm{H})$, 7.35-7.50 (m, 5H); ${ }^{13} \mathrm{C}$ NMR $\left(100 \mathrm{MHz}, \mathrm{CD}_{2} \mathrm{Cl}_{2}\right) \delta 23.7\left(\mathrm{CH}_{2}\right), 25.1\left(\mathrm{CH}_{2}\right), 27.3\left(\mathrm{CH}_{2}\right), 31.8$ $\left(\mathrm{CH}_{2}\right), 32.4\left(\mathrm{CH}_{2}\right), 54.1\left(\mathrm{CH}_{3}\right), 57.0(\mathrm{CH}), 91.0(\mathrm{C}), 91.5(\mathrm{C}), 126.4\left(2 \mathrm{x} \mathrm{CH}_{2}\right), 128.1(\mathrm{CH})$, $128.5\left(2 \mathrm{x} \mathrm{CH}_{2}\right), 138.0(\mathrm{C}), 211.3(\mathrm{C})$; LRMS (70 eV, EI): $\mathrm{m} / \mathrm{z}(\%): 232$ ([M-CO] $\left.{ }^{+}, 15\right), 189$ (52), 121 (100), 105 (85), 77 (45); HRMS (70 eV, EI) calcd for $\mathrm{C}_{15} \mathrm{H}_{20} \mathrm{O}_{2}$ [M-CO] $]^{+}: 232.1457$, found: 232.1456 .

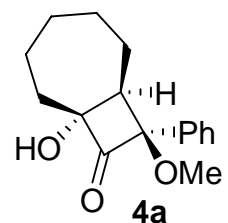

$\left(1 R^{*}, 8 R^{*}, 10 S^{*}\right)-8$-Hydroxy-10-methoxy-10-phenylbicyclo[6.2.0]decan-9-one

(4b). Colourless oil; m.p. $167-168{ }^{\circ} \mathrm{C} ; R_{\mathrm{f}} 0.23$ (hexane:AcOEt, 9:1); ${ }^{1} \mathrm{H}$ NMR $\left(400 \mathrm{MHz}, \mathrm{CDCl}_{3}\right) \delta$ $1.19-1.23(\mathrm{~m}, 1 \mathrm{H}), 1.37-1.44(\mathrm{~m}, 2 \mathrm{H}), 1.54-1.59(\mathrm{~m}, 1 \mathrm{H}), 1.62-1.68(\mathrm{~m}, 2 \mathrm{H}), 1.72-1.78(\mathrm{~m}$, $2 \mathrm{H}), 1.86-1.91(\mathrm{~m}, 2 \mathrm{H}), 1.96-1.99(\mathrm{~m}, 1 \mathrm{H}), 2.13-2.07(\mathrm{~m}, 1 \mathrm{H}), 2.24\left(\mathrm{dd},{ }^{3} J(\mathrm{H}, \mathrm{H})=11.6,2.4\right.$ 
$\mathrm{Hz}, 1 \mathrm{H}), 2.53(\mathrm{bs}, 1 \mathrm{H}), 2.52-2.59(\mathrm{~m}, 1 \mathrm{H}), 3.22(\mathrm{~s}, 3 \mathrm{H}), 7.33-7.39(\mathrm{~m}, 5 \mathrm{H}) ;{ }^{13} \mathrm{C} \mathrm{NMR}(100$ $\left.\mathrm{MHz}, \mathrm{CDCl}_{3}\right) \delta 20.8\left(\mathrm{CH}_{2}\right), 23.3\left(\mathrm{CH}_{2}\right), 23.9\left(\mathrm{CH}_{2}\right), 24.9\left(\mathrm{CH}_{2}\right), 27.3\left(\mathrm{CH}_{2}\right), 29.8\left(\mathrm{CH}_{2}\right), 54.6$ $\left(\mathrm{CH}_{3}\right), 56.6(\mathrm{CH}), 89.8(\mathrm{C}), 93.4(\mathrm{C}), 126.0\left(2 \mathrm{x} \mathrm{CH}_{2}\right), 128.1(\mathrm{CH}), 128.6\left(2 \mathrm{x} \mathrm{CH}_{2}\right), 137.5(\mathrm{C})$, 212.3 (C); LRMS (70 eV, EI): m/z (\%): 274 ([M] $\left.{ }^{+}, 3\right), 232$ ([M-CO $\left.]^{+}, 14\right), 189(57), 121(97)$, 105 (100), 77 (64); HRMS (70 eV, EI) calcd for $\mathrm{C}_{16} \mathrm{H}_{22} \mathrm{O}_{2}$ [M-CO] ${ }^{+}: 246.1614$, found: 246.1613 .

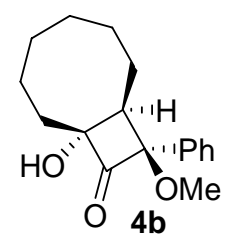

Synthesis 2-Butenolide 5. To a THF solution of cyclohexanone lithium enolate (2e, 1.2 mmol), prepared by method $B$ as above indicated, and cooled to $-78{ }^{\circ} \mathrm{C}$ was added carbene complex 1a $(1 \mathrm{mmol}, 312 \mathrm{mg})$ and THF $(10 \mathrm{~mL})$. The initial dark red solution of the starting carbene complex turned orange-yellow instantaneously at $-78^{\circ} \mathrm{C}$. The reaction mixture was stirred for $45 \mathrm{~min}$ while allowing the temperature to reach $-55^{\circ} \mathrm{C}$. It was then stirred for a further $60 \mathrm{~min}$ period at $-55^{\circ} \mathrm{C}$ to give a dark red-yellow mixture. The cool bath was removed and the reaction mixture was allowed to warm to room temperature and stirred for $30 \mathrm{~min}$. Then the reaction mixture was quenched with $\mathrm{H}_{2} \mathrm{O}(40 \mathrm{~mL})$ and neutralized with $2 \mathrm{~N} \mathrm{HCl}$. The resulting mixture was diluted with hexane/ethyl acetate, $10 / 1(110 \mathrm{~mL})$ and subjected to air oxidation under sunlight. After $24 \mathrm{~h}$ the brown suspension was filtered through Celite and extracted with diethyl ether $(3 \times 10 \mathrm{~mL})$. The organic layers were combined, dried over anhydrous $\mathrm{Na}_{2} \mathrm{SO}_{4}$ and concentrated in vacuo. The crude product was purified by flash column chromatography on silica gel (hexane/ethyl acetate, $5 / 1)$ to yield product $5(75 \%)$.

3-Phenyl-5,6,7,7a-tetrahydro-4H-benzofuran-2-one $\quad \mathbf{( 5 )}^{8} \quad$ Colourless $\quad$ oil; $\quad R_{\mathrm{f}} \quad 0.31$ (hexane:AcOEt, 3:1); IR (neat): $v_{\max }\left(\mathrm{cm}^{-1}\right)$ 2948, 2867, 1769, 1752, 1740, 1446, 1179; ${ }^{1} \mathrm{H}$ NMR $\left(300 \mathrm{MHz}, \mathrm{CDCl}_{3}\right) \delta 1.20-1.68(\mathrm{~m}, 3 \mathrm{H}), 1.90-2.03(\mathrm{~m}, 2 \mathrm{H}), 2.32(\mathrm{td}, J(\mathrm{H}, \mathrm{H})=13.6,5.6$ $\mathrm{Hz}, 1 \mathrm{H}), 2.54-2.62(\mathrm{~m}, 1 \mathrm{H}), 3.11$ (apparent dquintet, $J(\mathrm{H}, \mathrm{H})=14.2,2.0 \mathrm{~Hz}, 1 \mathrm{H}), 4.75$ (dd, $J(\mathrm{H}, \mathrm{H})=11.2,6.1 \mathrm{~Hz}, 1 \mathrm{H}), 7.34-7.47(\mathrm{~m}, 5 \mathrm{H}) ;{ }^{13} \mathrm{C} \mathrm{NMR}\left(75 \mathrm{MHz}, \mathrm{CDCl}_{3}\right) \delta 22.6\left(\mathrm{CH}_{2}\right), 26.3$ $\left(\mathrm{CH}_{2}\right), 26.8\left(\mathrm{CH}_{2}\right), 34.2\left(\mathrm{CH}_{2}\right), 79.8(\mathrm{CH}), 123.4(\mathrm{C}), 128.2(\mathrm{CH}), 128.4(2 \mathrm{xCH}), 128,8$ $(2 \times C H), 129.7(\mathrm{C}), 164.0(\mathrm{C}), 172.5(\mathrm{C})$; LRMS (70 eV, EI): m/z (\%): $214\left([\mathrm{M}]^{+}, 1\right), 213(32)$, 212 (100), 184 (35), 183 (70), 156 (68), 155 (80), 141 (78), 129 (48), 128 (91), 115 (99); HRMS (70 eV, EI): calcd for $\mathrm{C}_{14} \mathrm{H}_{14} \mathrm{O}_{2}[\mathrm{M}]^{+}: 214.0994$, found: 214.0994 . Elemental analysis calcd (\%) for $\mathrm{C}_{14} \mathrm{H}_{14} \mathrm{O}_{2}$ : C 78.28, H 6.59; found: C 78.29, H 6.57.

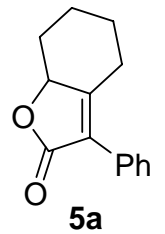

General Procedure for the Synthesis of 3,5-Dinitrobenzoyl Derivatives 6. ${ }^{9}$ To a solution of pure cyclopropanol 3c $(0.74 \mathrm{mmol}, 172 \mathrm{mg})$ or crude cyclopropanol 3d (310 $\mathrm{mg}$, obtained from $1 \mathrm{mmol}$ of complex 1a) in THF $(10 \mathrm{~mL})$ was successively added at room temperature 3,5-dinitrobenzoyl chloride ( $2 \mathrm{mmol}, 461 \mathrm{mg}), \mathrm{Et}_{3} \mathrm{~N}(4 \mathrm{mmol}, 562 \mu \mathrm{L})$, and 4-

\footnotetext{
${ }^{8}$ Demnitz, F. W. J. Tetrahedron Lett. 1989, 30, 6109-6112.

9 Tamura, N.; Mita, N.; Okabe, T.; Yamaguchi, T.; Fukushima, C.; Yamashita, M.; Morita, Y.; Morita, N.; Ishibashi, H.; Sakamoto, M. J. Org. Chem. 2001, 66, 2602-2610.
} 
dimethylaminopyridine $(0.1 \mathrm{mmol}, 20 \mathrm{mg})$. After stirring at RT for $50 \mathrm{~h}$, the reaction was quenched with $\mathrm{NH}_{4} \mathrm{Cl}(10 \mathrm{~mL}$, saturated aqueous solution) and THF was removed in vacuo. $\mathrm{CH}_{2} \mathrm{Cl}_{2}(10 \mathrm{~mL})$ was added, the layers were separated, and the aqueous layer was extracted with $\mathrm{CH}_{2} \mathrm{Cl}_{2}(3 \times 10 \mathrm{~mL})$. The organic layers were combined, dried over anhydrous $\mathrm{Na}_{2} \mathrm{SO}_{4}$ and concentrated in vacuo. The crude products were purified by flash column chromatography on silica gel (hexane/ethyl acetate, 9/1) to yield 6c (67\%) and 6d (72\%, based on complex 1a).

$\left(1 R^{*}, 7 S^{*}, 8 R^{*}\right)-8$-Methoxy-8-phenylbicyclo[5.1.0]octan-1-yl 3,5-dinitrobenzoate (6c). Colourless solid; m.p 155-157 ${ }^{\circ} \mathrm{C} ; R_{\mathrm{f}} 0.66$ (hexane:AcOEt, $\left.3: 1\right) ;{ }^{1} \mathrm{H}$ NMR $\left(300 \mathrm{MHz}, \mathrm{CDCl}_{3}\right.$ ) $\delta$ 0.90-1.19 (m, 3H), $1.59(\mathrm{~d}, J(\mathrm{H}, \mathrm{H})=11.4 \mathrm{~Hz}, 1 \mathrm{H}), 1.71-1.73(\mathrm{~m}, 2 \mathrm{H}), 1.84-1.94(\mathrm{~m}, 3 \mathrm{H})$, $2.41\left(\mathrm{dt},{ }^{2} \mathrm{~J}(\mathrm{H}, \mathrm{H})=13.4 \mathrm{~Hz},{ }^{3} \mathrm{~J}(\mathrm{H}, \mathrm{H})=6.4 \mathrm{~Hz}, 1 \mathrm{H}\right), 2.75\left(\mathrm{dd},{ }^{2} \mathrm{~J}(\mathrm{H}, \mathrm{H})=12.8 \mathrm{~Hz},{ }^{3} \mathrm{~J}(\mathrm{H}, \mathrm{H})=2.5\right.$ $\mathrm{Hz}, 1 \mathrm{H}), 3.12(\mathrm{~s}, 3 \mathrm{H}), 7.30-7.54(\mathrm{~m}, 3 \mathrm{H}), 7.64\left(\mathrm{~d},{ }^{3} \mathrm{~J}(\mathrm{H}, \mathrm{H})=6.9 \mathrm{~Hz}, 2 \mathrm{H}\right), 9.26(\mathrm{~s}, 3 \mathrm{H}) ;{ }^{13} \mathrm{C}$ NMR $\left(75 \mathrm{MHz}, \mathrm{CDCl}_{3}\right) \delta 25.9\left(\mathrm{CH}_{2}\right), 28.9\left(\mathrm{CH}_{2}\right), 29.0\left(\mathrm{CH}_{2}\right), 31.4\left(\mathrm{CH}_{2}\right), 31.6\left(\mathrm{CH}_{2}\right), 33.1$ $(\mathrm{CH}), 54.6\left(\mathrm{CH}_{3}\right), 72.9(\mathrm{C}), 74.0(\mathrm{C}), 122.2(\mathrm{CH}), 128.5(2 \times \mathrm{CH}), 128.6(\mathrm{CH}), 129.6(2 \times \mathrm{CH})$, $132.0(2 \mathrm{xCH}), 133.2(\mathrm{C}), 134.6(\mathrm{C}), 148.7$ (C), 163.1 (C); LRMS (70 eV, EI): m/z (\%): 426 $\left([\mathrm{M}]^{+}, 2\right), 231$ (27), $214(100), 105$ (72), 77 (46); HRMS (70 eV, EI) calcd for $\mathrm{C}_{22} \mathrm{H}_{22} \mathrm{O}_{7} \mathrm{~N}_{2}$ $[\mathrm{M}]^{+}:$426.1421, found: 426.1418. Elemental analysis calcd (\%) for $\mathrm{C}_{22} \mathrm{H}_{22} \mathrm{O}_{7} \mathrm{~N}_{2}: \mathrm{C} 61.97, \mathrm{H}$ 5.20, N 6.57; found: C 61.90, H 5.18, N 6.50.

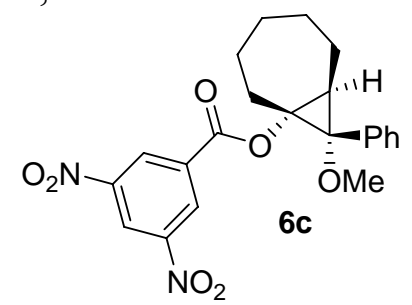

$\left(1 R^{*}, 8 S^{*}, 9 R^{*}\right)-9-M e t h o x y-9-p h e n y l b i c y c l o[6.1 .0]$ nonan-1-yl $\quad 3,5$-dinitrobenzoate $(6 \mathrm{~d})$. Colourless solid; m.p $168-170{ }^{\circ} \mathrm{C} ; R_{\mathrm{f}} 0.72$ (hexane:AcOEt, 3:1); ${ }^{1} \mathrm{H}$ NMR $\left(400 \mathrm{MHz}, \mathrm{CDCl}_{3}\right)$ $\delta$ 0.85-1.02 (m, 2H), 1.37-1.44 (m, 2H), 1.46-1.55 (m, 3H), $2.36\left(\mathrm{dq},{ }^{2} J(\mathrm{H}, \mathrm{H})=14.5 \mathrm{~Hz}\right.$, $\left.{ }^{3} \mathrm{~J}(\mathrm{H}, \mathrm{H})=3.4 \mathrm{~Hz}, 1 \mathrm{H}\right), 2.79\left(\mathrm{dt},{ }^{2} \mathrm{~J}(\mathrm{H}, \mathrm{H})=15.4 \mathrm{~Hz},{ }^{3} \mathrm{~J}(\mathrm{H}, \mathrm{H})=3.4 \mathrm{~Hz}, 1 \mathrm{H}\right), 3.11(\mathrm{~s}, 3 \mathrm{H}), 7.39-$ $7.46(\mathrm{~m}, 3 \mathrm{H}), 7.64\left(\mathrm{~d},{ }^{3} \mathrm{~J}(\mathrm{H}, \mathrm{H})=8.3 \mathrm{~Hz}, 2 \mathrm{H}\right), 9.22\left(\mathrm{~d},{ }^{4} \mathrm{~J}(\mathrm{H}, \mathrm{H})=2.1 \mathrm{~Hz}, 2 \mathrm{H}\right), 9.24(\mathrm{~m}, 1 \mathrm{H}) ;{ }^{13} \mathrm{C}$ $\operatorname{NMR}\left(100 \mathrm{MHz}, \mathrm{CDCl}_{3}\right) \delta 24.7\left(\mathrm{CH}_{2}\right), 25.9\left(\mathrm{CH}_{2}\right), 26.0\left(\mathrm{CH}_{2}\right), 26.4\left(\mathrm{CH}_{2}\right), 26.8\left(\mathrm{CH}_{2}\right), 28.9$ $\left(\mathrm{CH}_{2}\right), 32.6(\mathrm{CH}), 54.4\left(\mathrm{CH}_{3}\right), 68.8(\mathrm{C}), 70.3(\mathrm{C}), 122.2(\mathrm{CH}), 128.3(2 \mathrm{xCH}), 128.4(\mathrm{CH})$, $129.5(2 \times C H), 131.5(2 \times C H), 133.8(\mathrm{C}), 134.4(\mathrm{C}), 148.7$ (C), 162.8 (C); LRMS (70 eV, EI): m/z (\%): 440 ([M] $\left.]^{+}, 5\right), 245$ (86), 228 (100), 105 (82), 77 (64); HRMS (70 eV, EI) calcd for $\mathrm{C}_{23} \mathrm{H}_{24} \mathrm{O}_{7} \mathrm{~N}_{2}[\mathrm{M}]^{+}: 440.1578$, found: 440.1579 . Elemental analysis calcd (\%) for $\mathrm{C}_{23} \mathrm{H}_{24} \mathrm{O}_{7} \mathrm{~N}_{2}$ : C 62.72, H 5.49, N 6.36; found: C 62.70, H 5.50, N 6.30.<smiles>CO[C@@H]1[C@@H]2CCCCC[C@H]1[C@@]2(OC(=O)c1cc([N+](=O)[O-])cc([N+](=O)[O-])c1)c1ccccc1</smiles>

General Procedure for the Synthesis of 1,2-Dimethoxycyclopropanes 7. To a THF solution of the corresponding lithium enolate $\mathbf{2 a , b , f , g}(1.2 \mathrm{mmol})$, prepared by method $A$ or $B$ as above indicated (see also Table 2), and cooled to $-78^{\circ} \mathrm{C}$ was added the appropiate carbene complex 1a-c $(1 \mathrm{mmol})$ and THF $(10 \mathrm{~mL})$. After $5 \mathrm{~min}$, the cool bath was removed and the reaction mixture was allowed to warm to room temperature and stirred for a further 45 min period. The initial dark red solution of the starting carbene complex turned orange-yellow gradually when the cool bath was removed and it turned dark red-yellow at room temperature. The resultant mixture was concentrated in high vacuum, and the residue dissolved in $\mathrm{Et}_{2} \mathrm{O}(10$ 
$\mathrm{mL})$ and cooled to $-78{ }^{\circ} \mathrm{C}$. MeOTf $(2.0 \mathrm{mmol}, 224 \mu \mathrm{L})$ was added dropwise to the reaction. After $5 \mathrm{~min}$, the cool bath was removed and the reaction mixture was stirred for 30 min while allowing the temperature to reach room temperature. The reaction mixture was quenched with $\mathrm{NH}_{4} \mathrm{Cl}(20 \mathrm{~mL}$, saturated aqueous solution) and the resulting mixture was diluted with hexane/ethyl acetate, $10 / 1(110 \mathrm{~mL})$ and subjected to air oxidation under sunlight. After 2-12 $\mathrm{h}$ the yellow suspension was filtered through Celite and extracted with diethyl ether $(3 \times 10$ $\mathrm{mL}$ ). The organic layers were combined, dried over anhydrous $\mathrm{Na}_{2} \mathrm{SO}_{4}$ and concentrated in vacuo. The crude products were purified by flash column chromatography on silica gel (hexane/ethyl acetate, 20/1) to yield 7b (84\%), 7c (77\%), and 7e (73\%).

In two experiments the reaction mixture of carbene complex 1a,b and lithium enolate 2a prepared at $-78{ }^{\circ} \mathrm{C}$ as above described was stirred for $10 \mathrm{~min}$ while allowing the temperature to reach $-55^{\circ} \mathrm{C}$ and then stirred for a further $60 \min$ period at $-55^{\circ} \mathrm{C}$. The cool bath was removed and the reaction mixture was allowed to get room temperature and stirred for 30 min. The resultant mixture was concentrated, dissolved in $\mathrm{Et}_{2} \mathrm{O}$ and treated with MeOTf at $78{ }^{\circ} \mathrm{C}$ as above described to give, after work up, diastereomerically pure 1,2dimethoxycyclopropanes 7a (81\%) and 7d (81\%).

$\left(1 S^{*}, 2 S^{*}, 3 R^{*}\right)$-3-Ethyl-1,2-dimethoxy-1-phenyl-2-propylcyclopropane (7a). Colourless oil; $R_{\mathrm{f}} 0.45$ (hexane:AcOEt, 9:1); ${ }^{1} \mathrm{H}$ NMR $\left(300 \mathrm{MHz}, \mathrm{CDCl}_{3}\right) \delta 0.36\left(\mathrm{ddd},{ }^{2} J(\mathrm{H}, \mathrm{H})=14.1 \mathrm{~Hz}\right.$, $\left.{ }^{3} J(\mathrm{H}, \mathrm{H})=11.1,5.6 \mathrm{~Hz}, 1 \mathrm{H}\right), 0.90\left(\mathrm{t},{ }^{3} J(\mathrm{H}, \mathrm{H})=7.4 \mathrm{~Hz}, 3 \mathrm{H}\right), 1.18\left(\mathrm{dd},{ }^{3} J(\mathrm{H}, \mathrm{H})=9.0,5.1 \mathrm{~Hz}\right.$, $1 \mathrm{H}), 1.33\left(\mathrm{t},{ }^{3} \mathrm{~J}(\mathrm{H}, \mathrm{H})=7.3 \mathrm{~Hz}, 3 \mathrm{H}\right), 1.39-1.53(\mathrm{~m}, 1 \mathrm{H}), 1.66-1.79(\mathrm{~m}, 1 \mathrm{H}), 1.83-1.98(\mathrm{~m}, 2 \mathrm{H})$, 2.10-2.22 (m, 1H), $3.15(\mathrm{~s}, 3 \mathrm{H}), 3.61(\mathrm{~s}, 3 \mathrm{H}), 7.20-7.39(\mathrm{~m}, 5 \mathrm{H}).) ;{ }^{13} \mathrm{C}$ NMR $(75 \mathrm{MHz}$, $\left.\mathrm{CDCl}_{3}\right) \delta 14.0\left(\mathrm{CH}_{3}\right), 14.7\left(\mathrm{CH}_{3}\right), 15.1\left(\mathrm{CH}_{2}\right), 18.2\left(\mathrm{CH}_{2}\right), 32.1(\mathrm{CH}), 33.0\left(\mathrm{CH}_{2}\right), 54.7\left(\mathrm{CH}_{3}\right)$, $55.3\left(\mathrm{CH}_{3}\right), 67.8(\mathrm{C}), 71.0(\mathrm{C}), 127.1(\mathrm{CH}), 128.1(2 \mathrm{xCH}), 129.1(2 \mathrm{xCH}), 138.4(\mathrm{C})$; LRMS (70 eV, EI): m/z (\%): 248 ([M] $\left.]^{+}, 5\right), 220$ (80), 219 (96), 205 (99), 187 (49), 105 (100), 77 (49); HRMS (70 eV, EI) calcd for $\mathrm{C}_{16} \mathrm{H}_{24} \mathrm{O}_{2}[\mathrm{M}]^{+}:$248.1776, found: 248.1771. Elemental analysis calcd (\%) for $\mathrm{C}_{16} \mathrm{H}_{24} \mathrm{O}_{2}$ : C 77.38, $\mathrm{H}$ 9.74; found: $\mathrm{C} 77.19, \mathrm{H} 9.67$.

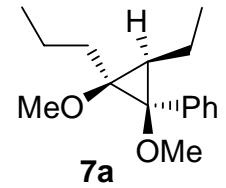

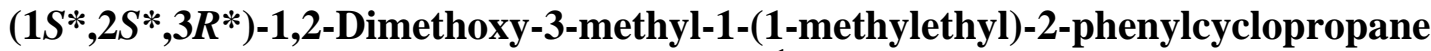

(7b). Colourless oil; $R_{\mathrm{f}} 0.63$ (hexane:AcOEt, 9:1); ${ }^{1} \mathrm{H}$ NMR $\left(300 \mathrm{MHz}, \mathrm{C}_{6} \mathrm{D}_{6}\right) \delta 0.92(\mathrm{~s}, 3 \mathrm{H}), 1.01$ $(\mathrm{m}, 1 \mathrm{H}), 1.03(\mathrm{~s}, 3 \mathrm{H}), 1.18\left(\mathrm{q},{ }^{3} J(\mathrm{H}, \mathrm{H})=6.3 \mathrm{~Hz}, 1 \mathrm{H}\right), 1.41\left(\mathrm{~d},{ }^{3} J(\mathrm{H}, \mathrm{H})=6.3 \mathrm{~Hz}, 3 \mathrm{H}\right), 3.04(\mathrm{~s}$, $3 \mathrm{H}), 3.80(\mathrm{~s}, 3 \mathrm{H}), 7.13-7.36(\mathrm{~m}, 5 \mathrm{H}) ;{ }^{13} \mathrm{C} \mathrm{NMR}\left(75 \mathrm{MHz}, \mathrm{CDCl}_{3}\right) \delta 7.0\left(\mathrm{CH}_{3}\right), 18.1\left(\mathrm{CH}_{3}\right)$, $18.8\left(\mathrm{CH}_{2}\right), 23.8\left(\mathrm{CH}_{2}\right), 33.9(\mathrm{CH}), 55.1\left(\mathrm{CH}_{3}\right), 59.6\left(\mathrm{CH}_{3}\right), 72.0(\mathrm{C}), 72.6(\mathrm{C}), 127.1(\mathrm{CH})$, $127.9(2 \mathrm{xCH}), 128.9(2 \mathrm{xCH}), 138.0(\mathrm{C})$; LRMS (70 eV, EI): $\mathrm{m} / \mathrm{z}(\%): 234\left([\mathrm{M}]^{+}, 4\right), 191(46)$, 105 (100), 77 (35); HRMS (70 eV, EI) calcd for $\mathrm{C}_{15} \mathrm{H}_{22} \mathrm{O}_{2}$ [M] $]^{+}: 234.1614$, found: 234.1613.

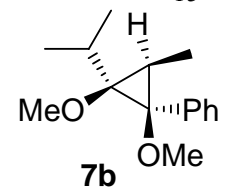

$\left(1 S^{*}, 2 S^{*}, 3 S^{*}\right)$-1,2-Dimethoxy-3-methyl-1-phenyl-2-(2-thienyl)cyclopropane

Colourless solid; m.p 101-103 ${ }^{\circ} \mathrm{C} ; R_{\mathrm{f}} 0.50$ (hexane:AcOEt, 9:1); ${ }^{1} \mathrm{H}$ NMR (400 MHz, $\mathrm{CDCl}_{3}$ ) $\delta 1.54\left(\mathrm{~d},{ }^{3} J(\mathrm{H}, \mathrm{H})=6.4 \mathrm{~Hz}, 3 \mathrm{H}\right), 2.12\left(\mathrm{q},{ }^{3} J(\mathrm{H}, \mathrm{H})=6.4 \mathrm{~Hz}, 1 \mathrm{H}\right), 3.26(\mathrm{~s}, 3 \mathrm{H}), 3.44(\mathrm{~s}, 3 \mathrm{H})$, $6.53\left(\mathrm{dd},{ }^{3} J(\mathrm{H}, \mathrm{H})=3.5 \mathrm{~Hz},{ }^{4} J(\mathrm{H}, \mathrm{H})=1.1 \mathrm{~Hz}, 1 \mathrm{H}\right), 6.70\left(\mathrm{dd},{ }^{3} J(\mathrm{H}, \mathrm{H})=5.0 \mathrm{~Hz},{ }^{4} J(\mathrm{H}, \mathrm{H})=3.6\right.$ $\mathrm{Hz}, 1 \mathrm{H}), 7.05\left(\mathrm{dd},{ }^{3} \mathrm{~J}(\mathrm{H}, \mathrm{H})=5.0 \mathrm{~Hz},{ }^{4} \mathrm{~J}(\mathrm{H}, \mathrm{H})=1.2 \mathrm{~Hz}, 1 \mathrm{H}\right), 7.16-7.27(\mathrm{~m}, 5 \mathrm{H}) ;{ }^{13} \mathrm{C} \mathrm{NMR}(100$ $\left.\mathrm{MHz}, \mathrm{CDCl}_{3}\right) \delta 6.6\left(\mathrm{CH}_{3}\right), 25.9(\mathrm{CH}), 55.7\left(\mathrm{CH}_{3}\right), 56.1\left(\mathrm{CH}_{3}\right), 72.0(\mathrm{C}), 67.5(\mathrm{C}), 72.0(\mathrm{C})$, $125.3(\mathrm{CH}), 125.9(\mathrm{CH}), 126.4(\mathrm{CH}), 127.4(\mathrm{CH}), 127.8(2 \times \mathrm{xH}), 128.9(2 \times \mathrm{CH}), 137.0(\mathrm{C})$, 143.1 (C); LRMS (70 eV, EI): m/z (\%): 274 ([M] $\left.]^{+}, 4\right), 259$ (20), 111 (100), 105 (86), 77 (35); HRMS (70 eV, EI) calcd for $\mathrm{C}_{16} \mathrm{H}_{18} \mathrm{O}_{2} \mathrm{~S}[\mathrm{M}]^{+}: 274.1022$, found: 274.1023 . 


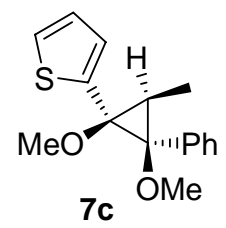

(1S*,2S*,3R*)-3-Ethyl-1,2-dimethoxy-1-(2-naphtyl)-2-propylcyclopropane

(7d). Colourless oil; $R_{\mathrm{f}} 0.78$ (hexane:AcOEt, 9:1); ${ }^{1} \mathrm{H}$ NMR $\left(300 \mathrm{MHz}, \mathrm{CDCl}_{3}\right) \delta 0.21$ (ddd, $\left.{ }^{2} J(\mathrm{H}, \mathrm{H})=14.7 \mathrm{~Hz},{ }^{3} \mathrm{~J}(\mathrm{H}, \mathrm{H})=10.9,5.5 \mathrm{~Hz}, 1 \mathrm{H}\right), 0.69\left(\mathrm{t},{ }^{3} \mathrm{~J}(\mathrm{H}, \mathrm{H})=7.5 \mathrm{~Hz}, 3 \mathrm{H}\right), 1.09(\mathrm{t}$, $\left.{ }^{3} \mathrm{~J}(\mathrm{H}, \mathrm{H})=7.3 \mathrm{~Hz}, 3 \mathrm{H}\right), 1.23\left(\mathrm{t},{ }^{3} \mathrm{~J}(\mathrm{H}, \mathrm{H})=7.0 \mathrm{~Hz}, 2 \mathrm{H}\right), 1.33-1.44(\mathrm{~m}, 1 \mathrm{H}), 1.65-1.78(\mathrm{~m}, 3 \mathrm{H})$, $3.06(\mathrm{~s}, 3 \mathrm{H}), 3.45(\mathrm{~s}, 3 \mathrm{H}), 7.39-7.49(\mathrm{~m}, 3 \mathrm{H}), 7.61(\mathrm{~s}, 1 \mathrm{H}), 7.75-7.83(\mathrm{~m}, 3 \mathrm{H}) ;{ }^{13} \mathrm{C}$ NMR $(75$ $\left.\mathrm{MHz}, \mathrm{CDCl}_{3}\right) \delta 14.1\left(\mathrm{CH}_{3}\right), 14.7\left(\mathrm{CH}_{3}\right), 15.2\left(\mathrm{CH}_{2}\right), 18.3\left(\mathrm{CH}_{2}\right), 32.2(\mathrm{CH}), 32.9\left(\mathrm{CH}_{2}\right), 54.8$ $\left(\mathrm{CH}_{3}\right), 55.5\left(\mathrm{CH}_{3}\right), 68.1(\mathrm{C}), 71.3(\mathrm{C}), 125.9(\mathrm{CH}), 126.1(\mathrm{CH}), 127.4(\mathrm{CH}), 127.5(\mathrm{CH})$, $127.8(\mathrm{CH}), 128.0(\mathrm{CH}), 132.7(\mathrm{C}), 133.1(\mathrm{C}), 136.2(\mathrm{C})$; LRMS (70 eV, EI): m/z (\%): 298 ([M] $\left.]^{+}, 11\right), 269$ (90), 155 (100), 127 (66); HRMS (70 eV, EI) calcd for $\mathrm{C}_{20} \mathrm{H}_{26} \mathrm{O}_{2}[\mathrm{M}]^{+}$: 298.1927, found: 298.1929 .

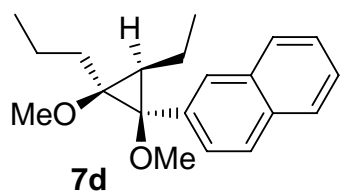

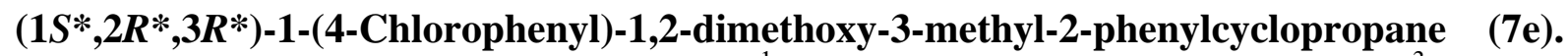
Colourless oil; $R_{\mathrm{f}} 0.26$ (hexane:AcOEt, 9:1); ${ }^{1} \mathrm{H}$ NMR $\left(300 \mathrm{MHz}, \mathrm{CDCl}_{3}\right) \delta 1.54\left(\mathrm{~d},{ }^{3} \mathrm{~J}(\mathrm{H}, \mathrm{H})=\right.$ $6.5 \mathrm{~Hz}, 3 \mathrm{H}), 2.13\left(\mathrm{q},{ }^{3} \mathrm{~J}(\mathrm{H}, \mathrm{H})=6.5 \mathrm{~Hz}, 1 \mathrm{H}\right), 3.26(\mathrm{~s}, 6 \mathrm{H}), 7.05-7.11(\mathrm{~m}$ with apparent $\mathrm{d}$ at $\left.7.05,{ }^{3} \mathrm{~J}(\mathrm{H}, \mathrm{H})=3.4 \mathrm{~Hz}, 10 \mathrm{H}\right) ;{ }^{13} \mathrm{C} \mathrm{NMR}\left(100 \mathrm{MHz}, \mathrm{CDCl}_{3}\right) \delta 6.55\left(\mathrm{CH}_{3}\right), 22.6(\mathrm{CH}), 55.7$ $\left(2 \mathrm{xCH}_{3}\right), 70.5(\mathrm{C}), 71.1(\mathrm{C}), 127.1(\mathrm{CH}), 127.8(4 \mathrm{xCH}), 128.6(2 \mathrm{xCH}), 129.9(2 \times \mathrm{CH}), 132.5$ (C), $136.0(\mathrm{C}), 136.8$ (C); LRMS (70 eV, EI): m/z (\%): 302 ([M] $\left.{ }^{+}, 10\right), 287$ (25), 139 (90), 105 (100), 77 (30); HRMS (70 eV, EI) calcd for $\mathrm{C}_{18} \mathrm{H}_{19} \mathrm{ClO}_{2}[\mathrm{M}]^{+}: 302.1068$, found: 302.1070 .

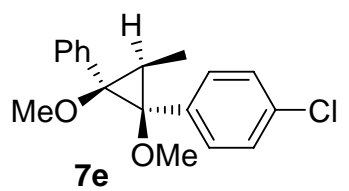

General Procedure for the Synthesis of 1-Methoxy-2-trimethylsilyloxycyclopropanes 8. To a THF solution of the corresponding lithium enolate $2 \mathbf{a}, \mathbf{b}, \mathbf{h}(1.2 \mathrm{mmol})$, prepared by method $B$ as above indicated (see also Table 2), and cooled to $-78{ }^{\circ} \mathrm{C}$ was added the appropiate carbene complex 1a,d $(1 \mathrm{mmol})$ and THF $(10 \mathrm{~mL})$. After $5 \mathrm{~min}$, the cool bath was removed and the reaction mixture was allowed to warm to room temperature and stirred for a further $45 \mathrm{~min}$ period. The initial dark red solution of the starting carbene complex turned orange-yellow gradually when the cool bath was removed and it turned dark red-yellow at room temperature. It was then concentrated in high vacuum, dissolved in THF $(10 \mathrm{~mL})$ and cooled to $-78^{\circ} \mathrm{C}$. TMSCl $(1.5 \mathrm{mmol}, 190 \mu \mathrm{L})$ was added dropwise to the reaction. After 5 min, the cool bath was removed and the reaction mixture was stirred for 30 min while allowing the temperature to reach room temperature. The reaction mixture was quenched with $\mathrm{NH}_{4} \mathrm{Cl}(20 \mathrm{~mL}$, saturated aqueous solution). The resulting mixture was diluted with hexane/ethyl acetate, 10/1 (110 mL) and subjected to air oxidation under sunlight. After $\sim 2 \mathrm{~h}$ the yellow suspension was filtered through Celite and extracted with diethyl ether $(3 \times 10$ $\mathrm{mL}$ ). The organic layers were combined, dried over anhydrous $\mathrm{Na}_{2} \mathrm{SO}_{4}$ and concentrated in vacuo. The crude products were purified by flash column chromatography on a short pad of silica gel (hexane/ethyl acetate, 200/1) to yield $\mathbf{8 a}(80 \%), \mathbf{8 b}(84 \%)$, and $\mathbf{8 c}(72 \%)$.

$\left(1 S^{*}, 2 S^{*}, 3 S^{*}\right)$-1-Ethyl-2-methoxy-3-methyl-2-phenyl-1-trimethylsilyloxycyclopropane

(8a). Light yellow oil; $R_{\mathrm{f}} 0.74$ (hexane:AcOEt, 10:1); ${ }^{1} \mathrm{H}$ NMR (300 MHz, $\mathrm{CDCl}_{3}$ ) $\delta 0.29$ (s, 
9H), 0.89-0.92 (m, 4H), 1.24-1.28 (m with apparent s at 1.25, 4H), $1.41\left(\mathrm{q},{ }^{3} \mathrm{~J}(\mathrm{H}, \mathrm{H})=7.3 \mathrm{~Hz}\right.$, $1 \mathrm{H}), 3.15(\mathrm{~s}, 3 \mathrm{H}), 7.29-7.42(\mathrm{~m}, 5 \mathrm{H}) ;{ }^{13} \mathrm{C} \mathrm{NMR}\left(75 \mathrm{MHz}, \mathrm{CDCl}_{3}\right) \delta 1.5\left(3 \mathrm{xCH}_{3}\right), 6.9\left(\mathrm{CH}_{3}\right)$, $9.4\left(\mathrm{CH}_{3}\right), 22.3(\mathrm{CH}), 29.7\left(\mathrm{CH}_{2}\right), 54.7\left(\mathrm{CH}_{3}\right), 66.1(\mathrm{C}), 69.7(\mathrm{C}), 127.0(\mathrm{CH}), 128.0(2 \times \mathrm{CH})$, $128.7(2 \mathrm{xCH}), 138.8$ (C); LRMS (70 eV, EI): m/z (\%): 278 ([M] $\left.]^{+}, 3\right), 263$ (16), 249 (62), 105 (100), 77 (24), 73 (44); HRMS (70 eV, EI) calcd for $\mathrm{C}_{16} \mathrm{H}_{26} \mathrm{O}_{2} \mathrm{Si}[\mathrm{M}]^{+}:$: 278.1696, found: 278.1697. Elemental analysis calcd (\%) for $\mathrm{C}_{16} \mathrm{H}_{26} \mathrm{O}_{2} \mathrm{Si}$ : C 69.01, H 9.01; found: C 69.28, H 9.11 .

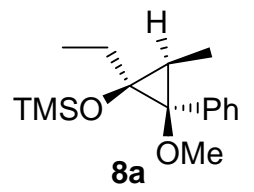

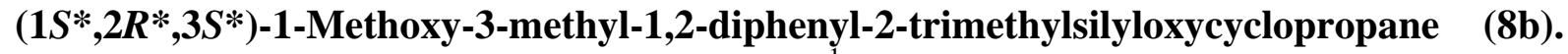
Light yellow oil; $R_{\mathrm{f}} 0.58$ (hexane:AcOEt, 10:1); ${ }^{1} \mathrm{H}$ NMR $\left(300 \mathrm{MHz}, \mathrm{CDCl}_{3}\right) \delta 0.12(\mathrm{~s}, 9 \mathrm{H})$, $1.48\left(\mathrm{~d},{ }^{3} J(\mathrm{H}, \mathrm{H})=6.4 \mathrm{~Hz}, 3 \mathrm{H}\right), 2.16\left(\mathrm{q},{ }^{3} \mathrm{~J}(\mathrm{H}, \mathrm{H})=6.2 \mathrm{~Hz}, 3 \mathrm{H}\right), 3.31(\mathrm{~s}, 1 \mathrm{H}), 7.09-7.16(\mathrm{~m}$, $10 \mathrm{H}) ;{ }^{13} \mathrm{C} \mathrm{NMR}\left(75 \mathrm{MHz}, \mathrm{CDCl}_{3}\right) \delta 1.5\left(3 \mathrm{xCH}_{3}\right), 7.4\left(\mathrm{CH}_{3}\right), 23.0(\mathrm{CH}), 55.5\left(\mathrm{CH}_{3}\right), 67.7(\mathrm{C})$, $70.1(\mathrm{C}), 126.6(\mathrm{CH}), 126.7(\mathrm{CH}), 127.4(2 \mathrm{xCH}), 127.7(2 \mathrm{xCH}), 128.5(4 \mathrm{xCH}), 137.8(\mathrm{C})$, 140.6 (C); LRMS (70 eV, EI): m/z (\%): 326 ([M] $\left.{ }^{+}, 4\right), 311$ (17), 105 (100), 77 (41), 73 (36); HRMS (70 eV, EI) calcd for $\mathrm{C}_{20} \mathrm{H}_{26} \mathrm{O}_{2} \mathrm{Si}[\mathrm{M}]^{+}:$326.1696, found: 326.1699. Elemental analysis calcd (\%) for $\mathrm{C}_{20} \mathrm{H}_{26} \mathrm{O}_{2} \mathrm{Si}$ : C 73.57, $\mathrm{H}$ 8.03; found: C 73.90, H 8.41.

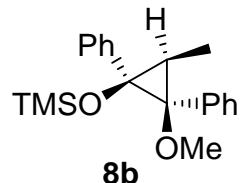

$\left(1 S^{*}, 2 S^{*}, 3 S^{*}\right)-3-E$ thyl-1-methoxy-2-propyl-1-(3-thienyl)-2-

trimethylsilyloxycyclopropane (8c). Light yellow oil; $R_{\mathrm{f}} 0.77$ (hexane:AcOEt, 9:1); ${ }^{1} \mathrm{H}$ NMR $\left(300 \mathrm{MHz}, \mathrm{CDCl}_{3}\right) \delta 0.21(\mathrm{~s}, 9 \mathrm{H}), 0.69-0.75(\mathrm{~m}, 1 \mathrm{H}), 0.84\left(\mathrm{t},{ }^{3} \mathrm{~J}(\mathrm{H}, \mathrm{H})=7.3 \mathrm{~Hz}, 3 \mathrm{H}\right)$, $1.01\left(\mathrm{dd},{ }^{3} J(\mathrm{H}, \mathrm{H})=7.8,6.0 \mathrm{~Hz}, 1 \mathrm{H}\right), 1.09\left(\mathrm{t},{ }^{3} J(\mathrm{H}, \mathrm{H})=7.4 \mathrm{~Hz}, 3 \mathrm{H}\right), 1.32-1.38(\mathrm{~m}, 1 \mathrm{H}), 1.40-$ $1.49(\mathrm{~m}, 1 \mathrm{H}), 1.56-1.65(\mathrm{~m}, 3 \mathrm{H}), 3.17(\mathrm{~s}, 3 \mathrm{H}), 7.07\left(\mathrm{dd},{ }^{4} \mathrm{~J}(\mathrm{H}, \mathrm{H})=2.9,1.2 \mathrm{~Hz}, 1 \mathrm{H}\right), 7.11(\mathrm{dd}$, $\left.{ }^{3} \mathrm{~J}(\mathrm{H}, \mathrm{H})=5.0,{ }^{4} \mathrm{~J}(\mathrm{H}, \mathrm{H})=1.2 \mathrm{~Hz}, 1 \mathrm{H}\right), 7.31\left(\mathrm{dd},{ }^{3} \mathrm{~J}(\mathrm{H}, \mathrm{H})=5.0,{ }^{4} \mathrm{~J}(\mathrm{H}, \mathrm{H})=2.9 \mathrm{~Hz}, 1 \mathrm{H}\right) ;{ }^{13} \mathrm{C} \mathrm{NMR}$ $\left(75 \mathrm{MHz}, \mathrm{CDCl}_{3}\right) \delta 1.3\left(3 \mathrm{xCH}_{3}\right), 14.2\left(\mathrm{CH}_{3}\right), 14.3\left(\mathrm{CH}_{3}\right), 15.5\left(\mathrm{CH}_{2}\right), 18.8\left(\mathrm{CH}_{2}\right), 32.1(\mathrm{CH})$, $54.9\left(\mathrm{CH}_{3}\right), 65.3(\mathrm{C}), 66.5(\mathrm{C}), 122.5(\mathrm{CH}), 125.3(\mathrm{CH}), 128.0(\mathrm{CH}), 141.6(\mathrm{C})$; LRMS (70 eV, EI): m/z (\%): 312 ([M] $\left.]^{+}, 6\right), 297$ (16), 283 (100), 111 (99), 73 (94), 71 (80); HRMS (70 eV, EI) calcd for $\mathrm{C}_{16} \mathrm{H}_{28} \mathrm{O}_{2} \mathrm{SSi}[\mathrm{M}]^{+}: 312.1574$, found: 312.1575 . Elemental analysis calcd (\%) for $\mathrm{C}_{16} \mathrm{H}_{28} \mathrm{O}_{2} \mathrm{SSi}$ : C 61.49, H 9.03; found: C 62.03, H 9.23.

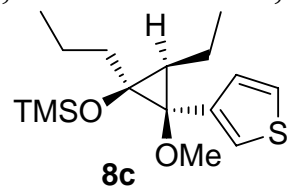

